\title{
Studies on the Chemical Constituents of Turpinia arguta Seem
}

\author{
Hongtao $\mathrm{Li}^{\mathrm{a}}$, Xiangming $\mathrm{Wu}^{\mathrm{b}}$, Fengxiang $\mathrm{Lin}^{\mathrm{c}}$, Yongming $\mathrm{Luo}^{\mathrm{d}_{*}}$ \\ Jiangxi University of Traditional Chinese Medicine, Nanchang, 330004, China \\ ae-mail: 756231347@qq.com, be-mail: 313198682@qq.com, ce-mail: 470623290 @qq.com, \\ ${ }^{d *}$ Corresponding author e-mail: loym999@126.com
}

Key words: Turpinia arguta Seem, chemical constituents, triterpenoids, flavonoids

\begin{abstract}
From the Turpinia arguta Seem, twelve compounds were isolated and identified as corosolic acidacid-28-O- $\beta$-D-glucopyranosyl ester(1), pomolic acid(2), ursolic acid(3), quercetin (4), rhoifolin (5), apigenin-7-O-rutinoside(6), quercetin-3- O-a-L-ara- binopyranoside(7), p-hydroxy cinnamic acid(8), caffeic acid(9), gallic acid, (10) 3,4-dihydroxybenzonic acid(11), phydroxybenzoic acid(12). Their structures were determined by ${ }^{1} \mathrm{H}-\mathrm{NMR},{ }^{13} \mathrm{C}-\mathrm{NMR}$ spectral data.
\end{abstract}

\section{Introduction}

Turpinia arguta Seem is distributed in the southern part of China. The leaves of Turpiniae arguta Seem has been used as an antibacterial, anti-inflammatory and analgesia agent in China for thousands.Flavonoids, megastigmanes and triterpenoids have been identified as constituents of the plant[1,2]. In the present paper, twelve compounds were isolated and identified as corosolic acidacid-28-O- $\beta$-D-glucopyranosyl ester(1), pomolic acid(2), ursolic acid(3), quercetin (4), rhoifolin (5), apigenin-7-O-rutinoside(6), quercetin-3- O-a-L-ara- binopyranoside(7), p-hydroxy cinnamic acid(8), caffeic acid(9), gallic acid, (10) 3, 4-dihydroxybenzonic acid(11), p-hydroxybenzoic acid(12) by means of ${ }^{1} \mathrm{H}-\mathrm{NMR},{ }^{13} \mathrm{C}-\mathrm{NMR}$ spectral data.

\section{Experimental}

General experimental procedures All melting points were determined on a X-4A micro-melting point apparatus and are uncorrected. IR spectra were recorded with an American Nicolet IMPACT-400 Fourier Transform Infrared Spectrometer. ${ }^{1} \mathrm{H}-\mathrm{NMR}$, ${ }^{13} \mathrm{C}-\mathrm{NMR}$, HSQC and HMBC were recorded on an American Varian company INOVA-400 and SYSTEM-600 FT NMR spectrometer. Silica gel (200-300 mesh; Qingdao Marine Chemical Plant) was used for column chromatography.

Plant material Turpinia arguta Seem were collected at Ganzhou, Jiangxi Province, China in September of 2013 and authenticated by Professor Q. Lui, Jiangxi University of Traditional Chinese Medicine. A voucher specimen (13-09-18) of the plant is deposited at the Herbarium of Jiangxi University of Traditional Chinese Medicine.

Extraction and isolation The leaves of Turpiniae arguta Seem was reduced to a coarse powder and refluxed with 95\% ethanol three times, After evaporation of ethanol in vacuo, the aqueous residue was diluted with water and was extracted with chloroform, ethyl acetate and n-butanol saturated with water to give the respective extracts after solvent removal. The ethyl acetate-solution portion(58g) was subjected to column chromatography on silica gel using a gradient mixture of 
chloroform-methanol (from 95:5 to 50:50) as eluting solvent to afford compound 2, 3, 4, 11, 12. The n-butanol-solution portion was evaporated, and the part extract(90g) was fractionated by silica gel column chromatography, eluted with $\mathrm{CHCl}_{3}-\mathrm{MeOH}-\mathrm{H}_{2} \mathrm{O}(6: 4: 1)$ to give five fractions (Fractions I-V). Fraction IIIwas subjected to a silica-gel column eluted with $\mathrm{CHCl}_{3}-\mathrm{MeOH}-\mathrm{H}_{2} \mathrm{O}$ (7:3:1) and preparative HPLC $\left[\mathrm{H}_{2} \mathrm{O}-\mathrm{MeOH}\right.$ (60:40), 5ml/min, monitored at $285 \mathrm{~nm}$ ] to give compound 5 10. Fraction V was subjected to preparative HPLC $\left[\mathrm{H}_{2} \mathrm{O}-\mathrm{CH}_{3} \mathrm{CN}\right.$ (70:30), 5ml/min, monitored at $210 \mathrm{~nm}$ ] to afford compound 1.

\section{Results and discussion}

Compound 1 was white powder, mp242 $244^{\circ} \mathrm{C} .{ }^{1} \mathrm{H}-\mathrm{NMR}\left(\mathrm{C}_{5} \mathrm{D}_{5} \mathrm{~N}, 400 \mathrm{MHz}\right) \delta$ : 0.96 (3H,d, $\mathrm{J}=6 \mathrm{~Hz}), 0.99$ (3H, d, J=6.2 Hz), 1.01 (3H, s), 1.05(3H, s), 1.07 (3H, s), 1.23 (3H, s), 1.28 (3H, s) , $3.36(1 \mathrm{H}, \mathrm{d}, \mathrm{J}=9 \mathrm{~Hz}), 4.15$ (1H,td, $\mathrm{J}=11,4 \mathrm{~Hz}), 5.44(1 \mathrm{H}, \mathrm{t}, \mathrm{J}=3 \mathrm{~Hz}) .{ }^{13} \mathrm{C}-\mathrm{NMR}\left(\mathrm{C}_{5} \mathrm{D}_{5} \mathrm{~N}, 100 \mathrm{MHz}\right) \delta$ : 48.1(C-1), 68.8(C-2), 83.1(C-3), 39.3(C-4), 55.2(C-5), 18.7(C-6), 33.1(C-7), 40.4(C-8), 48.2(C-9), 38.2(C-10), 23.8(C-11), 125.8(C-12), 139.4(C-13), 42.6(C-14), 28.1(C-15), 24.6(C-16), 48.1(C-17), 53.2(C-18), 39.5(C-19), 39.3(C-20), 31.8(C-21), 37.6(C-22), 29.6(C-23), 17.0(C-24), 17.2(C-25), 17.6(C-26), 23.6(C-27), 177.6(C-28), 17.2(C-29), 21.5 (C-30), 95.2(C-1'), 74.1(C-2'), 79.3(C-3’), 71.2(C-4'), 78.2(C-5'),62.3 (C-6'). On the basis of relevant reference [3] and its spectral data, its structure was elucidated to be corosolic acidacid-28-O- $\beta$-D-glucopyranosyl ester.

Compound 2 was white powder, mp: $298-300{ }^{\circ} \mathrm{C} .{ }^{1} \mathrm{H}-\mathrm{NMR}\left(\mathrm{CDCl}_{3}, 400 \mathrm{MHz}\right) \delta: 0.88(3 \mathrm{H}, \mathrm{s})$, $1.05(3 \mathrm{H}, \mathrm{d}, \mathrm{J}=6 \mathrm{~Hz}), 1.11(3 \mathrm{H}, \mathrm{s}), 1.16(3 \mathrm{H}, \mathrm{s}), 1.18(3 \mathrm{H}, \mathrm{s}), 1.37(3 \mathrm{H}, \mathrm{s}), 1.69(3 \mathrm{H}, \mathrm{s}), 3.25$ $(1 \mathrm{H}, \mathrm{dd}, \mathrm{J}=11,5 \mathrm{~Hz}), 5.59(1 \mathrm{H}, \mathrm{t}, \mathrm{J}=3 \mathrm{~Hz}) .{ }^{13} \mathrm{C}-\mathrm{NMR}\left(\mathrm{CDCl}_{3}, 100 \mathrm{MHz}\right) \delta: 39.7(\mathrm{C}-1), 26.4(\mathrm{C}-2)$, 78.0(C-3), 39.3(C-4), 56.3(C-5), 18.6(C-6), 33.3(C-7), 40.5(C-8), 47.4(C-9), 37.2(C-10), 23.9(C 11), 128.2(C-12), 139.1(C-13), 42.3(C-14), 29.4(C-15), 25.6(C-16), 48.8(C-17), 53.3(C-18), 72.5 (C-19), 42.2(C-20), 26.8(C-21), 37.6(C-22), 27.9(C-23), 17.3(C-24), 16.0(C-25), 17.0(C-26), 24.5 (C-27), 180.9(C-28), 27.3(C-29), 16.3 (C-30). On the basis of relevant reference [4] and its spectral data, its structure was elucidated to be pomolic acid.

Compound 3 was white powder, m p270 $271{ }^{\circ} \mathrm{C},{ }^{1} \mathrm{H} \operatorname{NMR}\left(\mathrm{C}_{5} \mathrm{D}_{5} \mathrm{~N}, 400 \mathrm{MHz}\right) \delta: 0.91$ (3H, s), 0.98 (3H, d, J=6.0 Hz), 1.02 (3H, d, J=6.4 Hz), 1.03 (3H, s), 1.07 (3H,s), 1.27 (3H, s), 1.29 (3H, s),3.42 (1H, dd, J=10,7Hz),5.54 (1H, t, J=3.2 Hz). ${ }^{13} \mathrm{C}-\mathrm{NMR}\left(\mathrm{C}_{5} \mathrm{D}_{5} \mathrm{~N}, 100 \mathrm{MHz}\right) \delta: 39.1(\mathrm{C}-1)$, 28.1(C-2), 78.1(C-3), 39.2(C-4), 55.3(C-5), 18.3(C-6), 33.3(C-7), 40.4(C-8), 48.1(C-9), 37.0(C-10), 23.7(C-11), 126.2(C-12), 139.3(C-13), 42.6(C-14), 28.8(C-15), 24.9(C-16), 48.0(C-17), 53.2(C-18), 39.5(C-19), 39.2(C-20), 31.8(C-21), 37.5(C-22), 27.9(C-23), 15.3(C-24), 16.0(C-25), 17.7(C-26), 23.5(C-27), 179.9(C-28), 17.3(C-29), 21.3 (C-30). On the basis of relevant reference [5] and its spectral data, its structure was elucidated to be ursolic acid.

Compound 4 was obtained as a yellow needle crystals, ,mp $311 \sim 313^{\circ} \mathrm{C},{ }^{1} \mathrm{H}$ NMR(DMSO- $d_{6}$, $400 \mathrm{MHz}) \delta: 12.47(1 \mathrm{H}, \mathrm{s}), 7.66(1 \mathrm{H}, \mathrm{d}, J=2.2 \mathrm{~Hz}), 7.52(1 \mathrm{H}, \mathrm{d}, J=7.8 \mathrm{~Hz}), 6.86(1 \mathrm{H}, \mathrm{d}, J=8.4 \mathrm{~Hz})$, $6.38(1 \mathrm{H}, \mathrm{d}, \mathrm{J}=2.0 \mathrm{~Hz}), 6.16(1 \mathrm{H}, \mathrm{d}, J=2.0 \mathrm{~Hz}) .{ }^{13} \mathrm{C}$ NMR(DMSO-d $\left.{ }_{6}, 100 \mathrm{MHz}\right) \delta: 175.7(\mathrm{C}-4)$, 164.4 (C-7), 160.6 (C-9), 156.1 (C-5), 147.7 (C-4'), 146.6 (C-2), 145.0 (C-3'), 135.6 (C-3), 121.9 (C-1'), 119.9 (C-6'), 115.5 (C-5'), 114.9 (C-2'), 102.7 (C-10), 98.3 (C-6), 93.3 (C-8). On the basis of spectral data[6], its structure was elucidated to be quercetin. 
Compound 5 were pale yellow granules, soluble in methanol, mp248-250 ${ }^{\circ} \mathrm{C},{ }^{1} \mathrm{H}$ NMR (DMSO $\left.-d_{6}, 400 \mathrm{MHz}\right) \delta: 12.96(1 \mathrm{H}, \mathrm{s}, \mathrm{OH}), 10.43(1 \mathrm{H}, \mathrm{s}, \mathrm{OH}), 7.94(2 \mathrm{H}, \mathrm{d}, \mathrm{J}=8.8 \mathrm{~Hz}, \mathrm{H}-2$ ', 6 '), $6.94(2 \mathrm{H}$, d, J = 8.8 Hz, H-3', 5'), 6.87 (1H, s, H-3 ), 6.79 (1H, d, J = 2.1 Hz, H-8), 6.38 (1H, d, J = 2.1 Hz, H-6). ${ }^{13}$ C-NMR (DMSO-d, 100 MHz) $\delta: 182.45$ (C-4), 164.74 (C-2), 161.86 (C-7), 161.58 (C-5), 157.45 (C-9 ), 129.05 (C-2 ', 6), 121.46 (C-1'), 116.50 (C-3', 5'), 105.89 (C-10), 103.71 (C-3), 100.93(C-1"), 98.27 (C-6), 98.32 (C-1"'), 94.98 (C-8), 77.68 (C-2"), 77.48 (C-3"), 76.72 (C- 5"), 72.33 (C-4"), 70.94 (C-4"'), 70.86 (C-3"'), 70.11 (C-2 "'), 68.81 (C-5"'), 60.93 (C-6"), 18.54 (C-6"'). The above data is consistent with the literatures, this compound is identified as rhoifolin.

Compound 6 was obtained as an amorphous solid. ${ }^{1} \mathrm{H}-\mathrm{NMR}\left(\mathrm{DMSO}-\mathrm{d}_{6}, 600 \mathrm{MHz}\right) \delta: 7.92(2 \mathrm{H}$, d, $J=9.0 \mathrm{~Hz}, \mathrm{H}-2^{\prime}$, H-6'), 6.92 (2H, d, J=9.0Hz, H-3', H-5' ) , 6.83 (1H,s), 6.76 (1H,d,J=2.4Hz,8-H）, 6.44(1H,d,J=2.4 Hz ,6-H). ${ }^{13} \mathrm{C}-\mathrm{NMR}\left(150 \mathrm{MHz}, \mathrm{DMSO}-d_{6}\right) \delta: 181.9$ (C-4),162.8 (C-5) ,161.1 (C-7),156.8 (C-9),128.6 (C-2',C-6') , 116.2 (C-3',C-5') ,105.3 (C-10) , 100.5 (C-1") , 99.8 (C-1"') , 99.4 (C-6) , 94.7 (C-8) , 76.2 (C-5") , 75.5 (C-3') , 73.0 (C-4"') , 72.0 (2") , 70.7 (C-4" ) , 70.3 (C-3"') , 69.5 (C-2"') , 68.2 (C-5"') , 66.0 (C-6") , 17.7 (C-6"') . On the basis of relevant reference [7] and its spectral data, its structure was elucidated to be apigenin- 7- Orutinoside.

Compound 7 was obtained as a yellow powder. ${ }^{1} \mathrm{H}-\mathrm{NMR}\left(\mathrm{DMSO}-\mathrm{d}_{6}, 600 \mathrm{MHz}\right) \delta:$ 7.64(1H,dd, $J=$ 9,2 Hz, H-6'), 7.50(1H,d,J= 1.8 Hz, H-2'), 6.83(1H,d, J= 8.4 Hz, H-5'), 6.38(1H, d, J=1.8Hz, H-8), 6.18(1H,d,J=1.2Hz, H-6), $5.26(1 \mathrm{H}, \mathrm{d}, J=5.4 \mathrm{~Hz}, \mathrm{H}-1 ") \quad .{ }^{13} \mathrm{C}-\mathrm{NMR}\left(\mathrm{DMSO}-d_{6}, 125 \mathrm{MHz}\right) \delta: 177.4$ (C-4) , 164.6 (C-7) , 161.1 (C-5) , 156.3 (C-9) , 156.1 (C-2) , 148.6 (C-4') , 145.0 (C-3’' ), $133.6(\mathrm{C}-3), 122.0\left(\mathrm{C}-6^{\prime}\right), 120.8\left(\mathrm{C}-1^{\prime}\right), 115.7\left(\mathrm{C}-5^{\prime}\right), 115.3\left(\mathrm{C}-2^{\prime}\right), 103.7$ (C-10), 101.4 (C-1”) , 98.7 (C-6) , 93.5 (C-8), 71.6 (C-2”) , 70.7 (C-3”) , 60.0 (C-4”) , 64.2 (C-5”) On the basis of spectral data[8], its structure was elucidated to be quercetin-3-O-a-L-arabinopyranoside.

Compound 8 was white powder, soluble in methanol, mp217-219 ${ }^{\circ} \mathrm{C} .{ }^{1} \mathrm{H}-\mathrm{NMR}$ (DMSO-d $\left.{ }_{6}, 400 \mathrm{MHz}\right) \delta: 12.15(1 \mathrm{H}, \mathrm{s}, \mathrm{OH}), 9.95(1 \mathrm{H}, \mathrm{s}, \mathrm{OH}), 6.28(1 \mathrm{H}, \mathrm{d}, \mathrm{J}=16 \mathrm{~Hz}, \mathrm{H}-3), 7.49(1 \mathrm{H}$, d, J = 16Hz, H-2), $6.78(2 \mathrm{H}, \mathrm{J}=8.8 \mathrm{~Hz}, \mathrm{H}-6,8), 7.50(2 \mathrm{H}, \mathrm{d}, \mathrm{J}=8.8 \mathrm{~Hz}, \mathrm{H}-5,9) .{ }^{13} \mathrm{C}-\mathrm{NMR}$ (DMSO-d $\left.{ }_{6}, 100 \mathrm{MHz}\right) \delta: 168.40$ (C-1), 160.05 (C-7), 144.63 (C-3), 130.55 (C-5,9), 125.73 (C-4), 116.20 (C-6,8), 115.79 (C-2). The above data is consistent with the literature, so it is identified as p-hydroxy cinnamic acid.

Compound 9 was obtained as a yellow powder, mp: 201-203 ${ }^{\circ} \mathrm{C},{ }^{1} \mathrm{H}-\mathrm{NMR}\left(\mathrm{CD}_{3} \mathrm{OD}, 600 \mathrm{MHz}\right)$ $\delta:$ 6.23(d, $J=15 \mathrm{~Hz}), 7.53(\mathrm{~d}, J=15 \mathrm{~Hz}), 6.73(\mathrm{~d}, J=8.4 \mathrm{~Hz}), 6.93(\mathrm{dd}, J=7.8 \mathrm{~Hz}, 1.8 \mathrm{~Hz}), 7.03(\mathrm{~d}, J$ $=1.8 \mathrm{~Hz}) .{ }^{13} \mathrm{C}-\mathrm{NMR}\left(\mathrm{CD}_{3} \mathrm{OD}, 150 \mathrm{MHz}\right) \quad \delta: 171.6(\mathrm{COOH}), 115.3$ (C-6) ,116.1 (C-5) ,117.0 (C-2) 122.9 (C-8), 128.1 (C-1),146.9 (C-3), 147.0 (C-8),149.5 (C-4) , 171.6 (C-9) . On the basis of relevant reference [9] and its spectral data, its structure was elucidated to be caffeic acid.

Compound 10 was yellow needle crystal, soluble in methanol, mp201-203 ${ }^{\circ} \mathrm{C},{ }^{1} \mathrm{H}-\mathrm{NMR}$ $\left(\mathrm{DMSO}_{6}, 400 \mathrm{MHz}\right) \delta: 12.22(1 \mathrm{H}, \mathrm{s}, \mathrm{OH}), 9.18(2 \mathrm{H}, \mathrm{s}, \mathrm{OH}), 8.92(1 \mathrm{H}, \mathrm{s}, \mathrm{OH}), 6.92(2 \mathrm{H}, \mathrm{s}$, $\mathrm{H}-2,6),{ }^{13} \mathrm{C}-\mathrm{NMR}$ (DMSO- $\left.d_{6}, 100 \mathrm{MHz}\right) \delta: 167.91$ (COOH), 145.86 (C-3,5), 138.44 (C-4), 120.89 (C-1), 109.17 (C-2,6). The above data is consistent with the literature, it is identified as gallic acid. 
Compound 11 was obtained as colorless needles, mp: 210 212 ${ }^{\circ} \mathrm{C}$. UV $\lambda_{\max }(\mathrm{MeOH}): 206 \mathrm{~nm}$ 254nm. IR ( KBr ) cm ${ }^{-1}$ : 3207, 1676, 1601, 1529, 1298, 1466, 1419, 1381, 1130, 1095, 941, 766, 638, 577. EI-MS m/z (\%): 154(M+85), 137(100), 119(3), 110(31), 97(5), 91(6), 81(22), 69(3), 63(32), 53(34), 44(35), 41(6). ${ }^{1} \mathrm{H}-\mathrm{NMR}\left(\mathrm{CD}_{3} \mathrm{OD}, 400 \mathrm{MHz}\right) \delta$ : $7.47(1 \mathrm{H}, \mathrm{s}, \mathrm{H}-2)$ 7.45(1H, d, H-6), 6. 82(1H, s, H-5) $.{ }^{13} \mathrm{C}-\mathrm{NMR}\left(\mathrm{CD}_{3} \mathrm{OD}, 100 \mathrm{MHz}\right) \delta: \quad 170.2,151.5(\mathrm{C}-4), 146.0(\mathrm{C}-3), 123.9(\mathrm{C}-1), 123.1(\mathrm{C}-6)$, 117.7(C-2), 115.7(C-5). On the basis of spectral data, its structure was elucidated to be 3 , 4-dihydroxybenzonic acid.

Compound 12 was white powder. ${ }^{1} \mathrm{H}-\mathrm{NMR}\left(\mathrm{CD}_{3} \mathrm{OD}, 400 \mathrm{MHz}\right) \delta: 7.86(2 \mathrm{H}, \mathrm{d}, J=8.4 \mathrm{~Hz})$, 6.82(2H, d, $J=8.4 \mathrm{~Hz}) .{ }^{13} \mathrm{C}-\mathrm{NMR}\left(\mathrm{CD}_{3} \mathrm{OD}, 100 \mathrm{MHz}\right) \delta: 163.4(\mathrm{C}-1), 132.7(\mathrm{C}-3$ ),116.2( C-2 ),123.1 (C-4) . On the basis of spectral data[10], its structure was elucidated to be p-hydroxybenzoic acid.

\section{Acknowledgments}

This work was supported by the 555 talent projects of Jiangxi province and Jiangxi University of Traditional Chinese Medicine.

\section{References}

[1]Fang W., Flora of China[M], Vol. 46, Beijing: Science Press, 1981,p.27-37.

[6] Sun J., Liu X., etc. Progress on Chemical Composition and Pharmacological Activity of Turpinia arguta Seem[J]. Food and Drug, 2011, 13(11):441-442

[3]Mahato B.S., Kundu P.A., ${ }^{13}$ C NMR spectra of pentacyclic triterpenoids A compilation and some salient features. Phytochemistry, 1994; 37, 1517-1575

[4] Cheng D L, Cao X P. Pomolic acid derivatives from the root of Sanguisorba officinalis[J]. Phytochemistry, 1992, 31(4): 1317-1320.

[5] Poehland B L, Carte B K, Francis T A, et al. In vitro antiviralactivity of dammar resin triterpenoids [J]. J Nat Prod, 1987, 50(4):706-713.

[6]Yu J., Qi Y., Zhu M., Studies on the Flavonoids of lllicium Jiadifengpi B. N. Chang [J]., Chin J Mod Appl Pharm, 2008, 25(3): 208-209.

[7] Tian Y., Liu X., Dong J., Apigenin glycosides from Euphorbia humifusa Wild. [J]. Acta Pharmaceutica Sinica, 2009, 44 (5) : 496-499.

[8] Shi S., Zou H., Zhang Y.,et al, Chemical constituents from Neo-Taraxacum siphonathum[J]. China Journal of Chinese Materia Medica,, 2009, 34 ( 8) : 1002-1004.

[9] Li H., Luo Y., He Z.. Chemical Consituents from Lonicera saccata[J]. Chin J Apol Eviron Biol, 2007, 13(2):188-191.

[10] Ma Y., Wang P., Chemical constituents of the leaves of Xanthoceras sorbifolia Bunge [J]. Chinese Traditional Patent Medicine, 2010, 32 (10): 1750-1753. 